\title{
Efficacy of Melatonin on Postoperative Outcomes after Hysterectomy: A Randomized, Double-blind, Placebo-controlled Trial
}

Prok Laosuwan ( $\square$ prok.l@chulahospital.org)

King Chulalongkorn Memorial Hospital https://orcid.org/0000-0003-4728-3557

Kamolwan Dechaworawut

King Chulalongkorn Memorial Hospital

Oraluxna Rodanant

Chulalongkorn University

\section{Somrat Charuluxananan}

Chulalongkorn University

Research article

Keywords: melatonin, hysterectomy, acute pain management, sleep quality, multimodal analgesia, postoperative pain

Posted Date: May 19th, 2020

DOl: https://doi.org/10.21203/rs.3.rs-26719/v1

License: (9) This work is licensed under a Creative Commons Attribution 4.0 International License. Read Full License 


\section{Abstract}

Background: Melatonin became a part of multimodal analgesia in several recent studies because of its analgesic, anxiolytic and anti-inflammatory properties. Incidence of anxiety and pain in patients who underwent hysterectomy are not low. Moreover, preoperative anxiety was related to postoperative pain. The hypothesis of this study was whether melatonin could improve pain and other postoperative conditions after hysterectomy.

Methods: A randomized, double-blinded, placebo-controlled trial recruitment of 54 patients, aged between 18 and 65 years old, planned to undergo hysterectomy, with or without oophorectomy under spinal anesthesia. The patients were allocated to receive $4 \mathrm{mg}$ prolonged-release melatonin at night before surgery and in the morning before surgery or 2 doses of placebo at the same time point. Morphine consumption within 24 hours with patient-controlled analgesia machine and visual analog scale (VAS) pain score were recorded. In addition, quality of sleep, Thai standard anxiety level score, fatigue, general well-being and satisfaction score were measured by a blinded assessor and analyzed.

Results: Mean of cumulative dose of morphine consumption in melatonin and placebo group were 33.04 \pm 10.42 and $42.63 \pm 8.21 \mathrm{mg}$, respectively. $(p<0.001)$ Mean of postoperative VAS pain scale was lower in the melatonin group at recovery room arrival (T0) (23.41 vs $8.07, p=0.01)$. However, there was not a significant difference between postoperative groups at 1(T1), 6 (T6) and $24 \mathrm{~h}$ (T24). Fatigue and general well-being score in the melatonin group were better than the placebo group.

Conclusion: Prolonged-release formulation of melatonin decreased pain intensity in post anesthetic care room and lowered doses of postoperative morphine within 24 hours after surgery. Postoperative fatigue, general well-being and satisfaction scores were better in the treatment group. However, there was no anxiety and sleep quality improvement. Melatonin may be an additional choice of multimodal analgesia for hysterectomy.

Clinical trial registration: TCTR20140516001, Registered 16 May, 2014, http://www.clinicaltrials.in.th/index.php? $\mathrm{tp}=$ regtrials\&menu=trialsearch\&smenu=fulltext\&task=search\&task2=view1\&id=1076, Efficacy of melatonin on postoperative outcomes after hysterectomy: a randomised, doubl-blinded, placebocontrolled trial

\section{Background}

A major problem after many surgical procedures is inadequate postoperative analgesia even after the multimodal analgesia concept was introduced. ${ }^{1}$ A national survey in the United States found that $80 \%$ of patients experienced acute pain after surgery. Most of these patients had moderate or severe pain. ${ }^{2}$ Uncontrollable postoperative pain is associated with respiratory function. ${ }^{3}$ In contrast, efficient pain management is related to positive physiologic effects in specific organs such as reduced 
thromboembolic or cardiac complications, including early recovery. ${ }^{4}$ After the gate control theory was proposed, a wider understanding of pain mechanisms was achieved. The last update of pain definition recognized that pain is a multidimensional and subjective distressing experience in terms of sensory, emotional, social or cognitive components. ${ }^{5,6}$

Uterine fibroids are the most common benign pelvic problem. Therefore, hysterectomy is a treatment option from a recent survey-based study in United States. The leading reasons for undergoing operation were healthcare provider recommendation, significant pain and distress. ${ }^{7}$ Common postoperative problems after hysterectomy are not only acute postoperative pain but also high preoperative anxiety levels. ${ }^{8}$ Diagnosis-related symptoms negatively result in physical and social activities including patients' quality of life. ${ }^{9}$ Preoperative anxiety in hysterectomy is strongly related to postoperative pain score as well. ${ }^{10,11}$ Benzodiazepine is a common medication as preoperative anxiolytic but may impair psychomotor performance.

Melatonin ( $\mathrm{N}$-acetyl-5-methoxytryptamine) is a pineal hormone regulating sleep-wake cycle in mammals. More than circadian rhythm stabilizing, exogenous melatonin has been investigated for other effects such as modulation of blood pressure, body temperature and cortisol control, immune function and antioxidative defense. ${ }^{12}$ Its strong chronobiotic properties and the ability to regulate circadian rhythm make melatonin a good choice for sleep disorders in the elderly. ${ }^{13}$ The exact analgesic mechanisms of melatonin are not known but may be involved with many receptors in brain. Involvement of $\beta$-endorphins, GABA receptor, opioid receptors and nitric oxide-arginine pathway were proposed. ${ }^{14}$ Several recent studies showed some benefits of perioperative melatonin in many aspects among different groups of patients

such as quality of recovery after surgery, diminished depressive symptoms and pain score reduction. ${ }^{15-17}$ However, melatonin's analgesic effect remains controversial in the perioperative period and requires more investigation. ${ }^{8}$ The hypothesis of the study was whether preoperative oral melatonin can potentiate the analgesic effects of intravenous morphine and improve sleep quality and anxiety levels. The aim of the present study was to examine the analgesic effects of oral long-acting melatonin premedication, compared to placebo, on morphine consumption in patients undergoing abdominal hysterectomy with or without oopherectomy. In addition, other therapeutic perspectives in clinical anesthesia such as anxiolytic effect, sleep quality, and also quality of life after hysterectomy were compared.

\section{Methods}

The present randomized, controlled and double-blinded study was conducted between April and December 2014 in King Chulalongkorn Memorial Hospital after approval of the Institutional Review Board of Faculty of Medicine, Chulalongkorn University (IRB No.428/56, COA No. 781/2013, Chairperson Associate Prof U. Jaisamrarn) on December 19, 2013. All written informed patient consents were obtained. Fifty-four patients, ASA physical status I-II and age 18-65 year scheduled for elective abdominal hysterectomy (with or without ovarian surgery) were enrolled into the randomised, double blind, placebo-controlled study. Patient exclusion criteria included history of heart disease, hepatic or 
renal failure, psychiatric disorders, sleep disorders, chronic pain syndromes, mental impairment, drug or alcohol abuse, patients receiving drugs with known analgesic and sedative properties, BMl over $30 \mathrm{~kg} / \mathrm{m}^{2}$ and patients who declined spinal anesthesia.

The patients were randomly divided into 2 groups ( 27 patients each) by using a random number table (1:1) and concealed opaque envelopes. These patients received either $4 \mathrm{mg}$ of prolonged-release formulation of oral melatonin (Circadin ${ }^{\circledR}$ ) (M group) or placebo (P group) at the night (8 PM) before the procedure and another dose 2 hours before surgery from a pharmacist who generated the random sequence and was not involved to the study. No other preoperative medication was given. Blinding and randomization were performed by an investigator who was not involved in patient evaluation. Other personnel involved in the patient's care were unaware of patient group assignment.

Preoperative visit was conducted the day before surgery. All patients were evaluated by the same anesthesia resident, who provided information on the preoperative course and instructed them on how to use the patient-control anesthesia (PCA) machine. Each patient was multidimensionally assessed; level of anxiety by the Amsterdam Preoperative Anxiety and Information Scale (APAIS) Thai version. ${ }^{18}$ Anxiety score ranged from 4-20 (anxiety score $>13$ possible to high level of anxiety up to 12 times). Sleep quality was measured by a questionnaire about subjective sleep quality using $100 \mathrm{~mm}$ VAS (visual analog scale; $0=$ best conceivable sleep and $100=$ worst conceivable sleep). Level of physical fatigue and general wellbeing were evaluated by using 10-point ordinal scale ( $1=$ least fatigue feeling and $10=$ most fatigue feeling and $100 \mathrm{~mm}$ VAS ( $0=$ extremely well and $100=$ extreme malaise), respectively, at the night before and 24 hours after the surgery.

Upon arrival in the operating room, all patients underwent standard monitoring. Before spinal anesthesia, $10 \mathrm{ml} / \mathrm{kg}$ of physiologic crystalloid solution was administered intravenously. Spinal anesthesia was performed by spinal needle at lumbar segment L2/3 or L3/4 with $0.5 \%$ hyperbaric bupivacaine $16-20 \mathrm{mg}$ according to attending anaesthesiologists. If any patient had anxiety or discomfort, continuous propofol $0.08-0.1 \mathrm{mg} / \mathrm{kg} / \mathrm{min}$ was given to maintain conscious sedation during the surgery. At the end of the surgery, sedation was stopped.

At recovery room, all patients received morphine via patient-controlled analgesia (PCA) machine; the PCA dose was $1 \mathrm{mg}$, a 6-min lockout and maximum dose of $30 \mathrm{mg}$ within 4 hours and no basal rate was applied. In the first 2-hour postoperative period, if the patients had VAS pain score more than $40 \mathrm{~mm}$ after being connected to morphine PCA, morphine $0.1 \mathrm{mg} / \mathrm{kg}$ was further injected. PCA pump was continued for 24 hours after surgery. Four mg of ondansetron every 6 hours was administered for nausea/vomiting as requirement. No other analgesic was allowed.

The primary outcome with respect to the efficacy of the study drug was postoperative morphine consumption in 24 hours. Secondary outcomes were postoperative pain score, anxiety, sleep quality, general well-being and satisfaction with pain treatment. Postoperative pain was assessed using $100 \mathrm{~mm}$ $\operatorname{VAS}(0=$ no pain and $100=$ worst imaginable pain $)$ when arriving at recovery room $(\mathrm{T} 0), 1(\mathrm{~T} 1), 6(\mathrm{~T} 6)$ and 
24(T24) hours. Satisfaction with pain treatment and nausea/vomiting were assessed using $100 \mathrm{~mm}$ VAS at $24 \mathrm{~h}$ postoperatively. Other adverse effects, surgical and anesthetic complications were recorded.

\section{Statistical analysis}

The sample size of 25 patients in each group was required to detect difference between groups in reduce postoperative morphine consumption $0.1 \mathrm{mg} / \mathrm{kg} / \mathrm{min}$ with a confidence level of $90 \%$ and a significance level of $5 \%$ according to data from the previous study. ${ }^{19}$ Twenty- seven patients were enrolled in each group, allowing a 10\% drop-out rate. Statistical analysis was calculated by using SPSS software version 17.0. Data are presented as mean \pm standard deviation unless stated otherwise. Comparison of morphine consumption was analyzed using unpaired t-test. Pain score, anxiety and sleep quality were analyzed using repeated measure ANOVA. Satisfaction and nausea/ vomiting were analyzed using Chi-squared test (Fisher's exact test if appropriate). A $p$-value $<0.05$ was considered statistically significant.

\section{Results}

Fifty-four patients were enrolled into the study. No patient was excluded from the study after enrollment. The patient characteristics in each group, 27 patients, including diagnosis and types of operations were comparable between both groups (as shown in Table 1). Doses of bupivacaine, propofol and ephedrine were similar. There was no significant difference in anesthetic level of bupivacaine and number of patients who required sedation. There was no statistical difference in surgical variables including operation time and amount of blood loss. The number of intraoperative events such as hypotension needed treatments, bradycardia (Heart rate $<60 / \mathrm{min}$ ). were comparable in both groups and shown in Table 2.

The patients in the $\mathrm{M}$ and $\mathrm{P}$ group who required cumulative dose of morphine consumption in $24 \mathrm{~h}$ were $33.04 \pm 10.42$ and $42.63 \pm 8.21 \mathrm{mg}$, respectively $(p<0.001)$. Postoperative VAS of pain was significantly lower in the $\mathrm{M}$ group at recovery room arrival (T0) (23.41 vs 8.07, $p=0.01)$. However, there was no significant difference of VAS pain score between groups at 1(T1), 6 (T6) and 24 hours (T24) postoperatively. Satisfaction with pain treatment in the $\mathrm{M}$ group was significantly higher than in the $\mathrm{P}$ group. (8.56 \pm 1.25 vs $7.78 \pm 1.50, p=0.02$ ). (Table 3 ) There was no significant difference between groups in preoperative and postoperative anxiety level. VAS scale for fatigue and general well-being scores in the first postoperative day were significantly lower in the $\mathrm{M}$ group compared with the placebo group. ( $3.30 \pm 2.22$ vs. $5.15 \pm 1.85, p=0.002$ and $31.59 \pm 24.14$ vs. $49.78 \pm 14.87, p=0.002$, respectively) However, subjective sleep quality was no significant difference between groups.

\section{Discussion}

The present study demonstrates that long-acting oral melatonin improved VAS pain score and reduced cumulative dose of PCA morphine consumption in 24 hours. These results were similar to the previous studies in other procedures, such as prostatectomy, ${ }^{20}$ dental surgery, ${ }^{15}$ hand surgery, ${ }^{21}$ cataract surgery 
under topical anesthesia ${ }^{22}$ and abdominal hysterectomy. ${ }^{19,23}$ In contrast, some studies failed to show the effectiveness of perioperative melatonin in terms of analgesic outcomes. ${ }^{24,25}$ The variation of dose, route and timing of melatonin administration might affect these individual results, which remain inconclusive even after systematic review were conducted. ${ }^{8,26}$ Caumo et al. revealed the analgesic effect of preoperative oral melatonin. Melatonin reduced pain scores on VAS scale within postoperative period of 48 hours and lowered morphine consumption for 24 hours after abdominal hysterectomy, compared to placebo. ${ }^{23}$ Such a study proposed that postoperative anxiolytic effect of melatonin treatment led to antinociceptive effect. ${ }^{23}$ Contrast to that study, this study could not show a significant difference of anxiolysis, as well as VAS pain score after immediate postoperative phase at post-anesthesia care unit arrival. The present study investigated a $4 \mathrm{mg}$ of prolonged-release formulation of melatonin (Circadin ${ }^{\circledR}$, Neurim Pharmaceuticals, Tel-Aviv, Israel). This was a lower dose than other previous studies as premedication for analgesic effect. Forms of melatonin in all previous studies might be a short acting formulation or higher doses. However, from general clinical practice, 2-mg dose once daily of prolongedrelease melatonin showed clinical benefits in terms of sleep quality and quality of life in patients aged 55 years and older without unexpected effects. ${ }^{27}$ The therapeutic indication of this novel formulation melatonin is primary insomnia in elderly due to long duration of action and safety profiles. ${ }^{8}$ Exogenous melatonin modulates via activation of the MT1 and/or MT2 melatonin receptors in the central nervous system. ${ }^{13,27}$ In addition, there were several in vitro studies which demonstrated that the anti-nociceptive effects of melatonin could be reversed by various mechanism such as flumazenil, naloxone, potassium or calcium ion-channel-blockers. ${ }^{12}$ The present study is the first clinical study of prolonged-release formulation in perioperative period. A recent study in patients who underwent orthognathic surgery showed that prophylactic oral melatonin significantly decreased pain, numbness perception and were also correlated to lower serum hydrogen peroxide but higher antioxidant enzyme levels. ${ }^{28}$

Patients with postoperative sleep disturbance can suffer from delirium, delayed recovery and pain. ${ }^{29}$ Correlation between pharmacologic sleep promotion and perioperative pain control are still controversial. ${ }^{30}$ The present study failed to demonstrate the improved postoperative sleep quality. Similar to a recent meta-analysis in cholecystectomy, melatonin interventions showed no substantial impact on sleep quality and pain score after 1 and 3 hours. ${ }^{31}$ However, Kirksey A. et al concluded melatonin did not have effect on subjective sleep assessment but improved sleep efficiency and sleep time by actigraphy wrist bracelet measurement. ${ }^{32}$

Acute postoperative pain after hysterectomy may be complicated by anxiety state and psychological factors. A qualitative systematic review demonstrated that anxiety was a significant predictor for postoperative pain. ${ }^{33}$ Such result was similar to another study in patients who underwent hysterectomy, in which preoperative anxiety was a positive predictor of immediate postoperative pain, pain on wards and also pain at home. ${ }^{10}$ Moreover, Pinto et al. showed that anxiety predicted pain intensity at 48 hours after hysterectomy and also mediated pain catastrophizing. ${ }^{6}$ In several clinical studies and systematic reviews, the outcome of preoperative melatonin administered to reduce preoperative anxiety was still 
controversial among varied population and doses. ${ }^{15,17,19,34}$ Whereas another systematic review from Cochrane database concluded melatonin can reduce preoperative anxiety at the same rate as standard medication with midazolam if it was given within appropriate timing. ${ }^{35}$ However, the present study could not exhibit the benefit of melatonin as an anxiolytic.

In addition, the concept of immune-pineal axis influencing postoperative pain in patients who underwent hysterectomy was proposed. There was an inverse correlation between tumor necrosis factor (TNF) and nocturnal melatonin level. Moreover, the lower melatonin level was accompanied by lower cortisol levels and patients required higher doses of analgesics. ${ }^{36}$ Therefore, exogenous melatonin might play a role for perioperative period especially in hysterectomy.

Fatigue has been defined as the lack of energy or exhaustion which is a complex, multifactorial symptom distinct from sleepiness or sadness. ${ }^{37}$ The incidence of postoperative fatigue following hysterectomy was frequent regardless of general or spinal anesthesia. ${ }^{38}$ Intensity of postoperative fatigue was the result of many biological factors, such as surgical stress response, anemia, declined nutritional status etc, psychological and social factors. ${ }^{38}$ Fatigue was associated to poor quality of life in cancer patients who underwent surgery. ${ }^{37}$ From the present study, melatonin enhanced subjective fatigue, general wellbeing VAS pain score and satisfaction score compared to placebo. These results were different from previous studies. Ivry M. et al. revealed melatonin improved quality of recovery following bariatric surgery in terms of sleep and pain levels. ${ }^{16}$ Although differing in definition and measurement, the present study demonstrated advantages of melatonin administration in early postoperative fatigue and recovery, but no improvement of sleep quality. This may be due to lower morphine requirement.

Limitations of this study include the quality of recovery questionnaire in Thai version, which was not validated at the time the study was conducted. Likert and VAS pain score were measured to represent overall subjective recovery condition. The details of each standard domain may be inconclusive. Second, the results were focused only on perioperative and acute postoperative periods. Future studies should evaluate the effect of melatonin on chronic pain after hysterectomy. Third, the present study revealed only benefits of preoperative 2 doses of $4 \mathrm{mg}$ of prolonged-release melatonin. Continuation of melatonin in postoperative period or earlier timing to load rather than one night before the surgery might be more appropriate with melatonin's pharmacokinetics and patient's metabolism. Moreover, to our knowledge, the appropriate dose and timing of oral prolonged-released melatonin was not established in perioperative period.

\section{Conclusion}

Preoperative orally prolonged-release melatonin had advantages in patients who underwent hysterectomy and/or oophorectomy under spinal anesthesia in terms of decreased morphine consumption, pain score in PACU. Postoperative fatigue, subjective general well-being, VAS pain score and also patients' satisfaction score in treatment group were better than placebo without adverse effects. 


\section{Abbreviations}

GABA; $ү$-aminobutyric acid

ASA; American Society of Anesthesiologists

VAS; Visual analog scale

APAIS; Amsterdam Preoperative Anxiety and Information Scale

PCA; patient-controlled analgesia

PACU; Post-anesthetic care unit

TNF; tumor necrosis factor

\section{Declarations}

Ethics approval and consent to participate: approval of the Institutional Review Board of Faculty of Medicine, Chulalongkorn University (IRB No.428/56, COA No. 781/2013, Chairperson Associate Prof U. Jaisamrarn) on December 19, 2013. Before study enrollment, all subjects reviewed and signed an informed consent document explaining the study procedures and potential risks.

Consent for publication: Not applicable

Availability of data and materials: The datasets used and/or analyzed during the current study are available from the corresponding author on reasonable request.

Competing interests: The authors declare that they have no competing interests

Funding: This study was supported by the Ratchadapisek Sompoch Endowment Fund, Faculty of Medicine, Chulalongkorn University, Thailand. The fund covered the expense of the all medications in the study.

Authors' contributions: PL helped with development of research idea, writing the proposal, data collection, data analysis, a major contribution in writing the manuscript. KD collected and analyzed the patient data. OR collected the patient data and reviewed the literature and perform final review of the manuscript. SC performed critical reviewing of research idea and the manuscript.

Acknowledgements: We appreciate the contributions to this work from the following: Administrators of Research Unit and all anesthesia personnel at post anesthesia care unit of the Department of Anesthesiology, King Chulalongkorn Memorial Hospital

Additional information: This study was presented at the $69^{\text {th }}$ Post Graduate Assembly in Anesthesiology (PGA) of the New York State Society of Anesthesiologists Inc., New York City, USA, on December 12, 2015 


\section{References}

1. Buvanendran A, Kroin JS. Multimodal analgesia for controlling acute postoperative pain. Current opinion in anesthesiology. 2009;22(5):588-93.

2. Apfelbaum JL, Chen C, Mehta SS, Gan TJ. Postoperative pain experience: results from a national survey suggest postoperative pain continues to be undermanaged. Anesthesia and analgesia. 2003;97(2):534-40, table of contents.

3. Beaussier M, Genty T, Lescot T, Aissou M. Influence of pain on postoperative ventilatory disturbances. Management and expected benefits. Annales francaises d'anaesthesie et de reanimation. 2014;33(78):484-6.

4. Kehlet $\mathrm{H}$. Acute pain control and accelerated postoperative surgical recovery. The Surgical clinics of North America. 1999;79(2):431-43.

5. Williams AC, Craig KD. Updating the definition of pain. Pain. 2016;157(11):2420-3.

6. Pinto PR, Mclntyre T, Almeida A, Araujo-Soares V. The mediating role of pain catastrophizing in the relationship between presurgical anxiety and acute postsurgical pain after hysterectomy. Pain. 2012;153(1):218-26.

7. Marsh EE, Al-Hendy A, Kappus D, Galitsky A, Stewart EA, Kerolous M. Burden, Prevalence, and Treatment of Uterine Fibroids: A Survey of U.S. Women. J Womens Health (Larchmt). 2018;27(11):135967.

8. Yousaf F, Seet E, Venkatraghavan L, Abrishami A, Chung F. Efficacy and safety of melatonin as an anxiolytic and analgesic in the perioperative period: a qualitative systematic review of randomised trials. Anesthesiology. 2010;113(4):968-76.

9. Downes E, Sikirica V, Gilabert-Estelles J, Bolge SC, Dodd SL, Maroulis C, et al. The burden of uterine fibroids in five European countries. Eur J Obstet Gynaecol Reprod Biol. 2010;152(1):96-102.

10. Kain ZN, Sevarino F, Alexander GM, Pincus S, Mayes LC. Preoperative anxiety and postoperative pain in women undergoing hysterectomy. A repeated-measures design. Journal of psychosomatic research. 2000;49(6):417-22.

11. Han C, Ge Z, Jiang W, Zhao H, Ma T. Incidence and risk factors of chronic pain following hysterectomy among Southern Jiangsu Chinese Women. BMC Anaesthesiol. 2017;17(1):103.

12. Andersen LP. The analgesic effects of exogenous melatonin in humans. Acta anaesthesiologica Scandinavica. 2016;60(7):1024-5. 
13. Liu J, Clough SJ, Hutchinson AJ, Adamah-Biassi EB, Popovska-Gorevski M, Dubocovich ML. MT1 and MT2 Melatonin Receptors: A Therapeutic Perspective. Annual review of pharmacology and toxicology. 2016;56:361-83.

14. Chen WW, Zhang X, Huang WJ. Pain control by melatonin: Physiological and pharmacological effects. Exp Ther Med. 2016;12(4):1963-8.

15. Seet E, Liaw CM, Tay S, Su C. Melatonin premedication versus placebo in wisdom teeth extraction: a randomised controlled trial. Singapore medical journal. 2015;56(12):666-71.

16. Ivry M, Goitein D, Welly W, Berkenstadt H. Melatonin premedication improves quality of recovery following bariatric surgery - a double blind placebo controlled prospective study. Surg Obes Relat Dis. 2017;13(3):502-6.

17. Hansen MV, Andersen LT, Madsen MT, Hageman I, Rasmussen LS, Bokmand S, et al. Effect of melatonin on depressive symptoms and anxiety in patients undergoing breast cancer surgery: a randomised, double-blind, placebo-controlled trial. Breast Cancer Res Treat. 2014;145(3):683-95.

18. Kunthonluxamee A, Pitimana-aree S, Laurujisawat P. Validity and Reliability of the Amsterdam Preoperative Anxiety and Information Scale (APAIS); Thai version in adult Thai pre-operative patients. Journal of the Psychiatric Association of Thailand. 2009;54(1):83-92.

19. Caumo W, Torres F, Moreira NL, Jr., Auzani JA, Monteiro CA, Londero G, et al. The clinical impact of preoperative melatonin on postoperative outcomes in patients undergoing abdominal hysterectomy. Anesthesia and analgesia. 2007;105(5):1263-71, table of contents.

20. Borazan H, Tuncer S, Yalcin N, Erol A, Otelcioglu S. Effects of preoperative oral melatonin medication on postoperative analgesia, sleep quality, and sedation in patients undergoing elective prostatectomy: a randomised clinical trial. Journal of anesthesia. 2010;24(2):155-60.

21. Mowafi HA, Ismail SA. Melatonin improves tourniquet tolerance and enhances postoperative analgesia in patients receiving intravenous regional anesthesia. Anesthesia and analgesia. 2008;107(4):1422-6.

22. Ismail SA, Mowafi HA. Melatonin provides anxiolysis, enhances analgesia, decreases intraocular pressure, and promotes better operating conditions during cataract surgery under topical anesthesia. Anesthesia and analgesia. 2009;108(4):1146-51.

23. Caumo W, Levandovski R, Hidalgo MP. Preoperative anxiolytic effect of melatonin and clonidine on postoperative pain and morphine consumption in patients undergoing abdominal hysterectomy: a double-blind, randomised, placebo-controlled study. The journal of pain : official journal of the American Pain Society. 2009;10(1):100-8. 
24. Andersen LP, Kucukakin B, Werner MU, Rosenberg J, Gogenur I. Absence of analgesic effect of intravenous melatonin administration during daytime after laparoscopic cholecystectomy: a randomised trial. Journal of clinical anesthesia. 2014;26(7):545-50.

25. Naguib M, Samarkandi AH. The comparative dose-response effects of melatonin and midazolam for premedication of adult patients: a double-blinded, placebo-controlled study. Anesthesia and analgesia. 2000;91(2):473-9.

26. Andersen LP, Werner MU, Rosenberg J, Gogenur I. A systematic review of peri-operative melatonin. Anesthesia. 2014;69(10):1163-71.

27. Lemoine P, Zisapel N. Prolonged-release formulation of melatonin (Circadin) for the treatment of insomnia. Expert opinion on pharmacotherapy. 2012;13(6):895-905.

28. Lee TYC, Curtin JP. The effects of melatonin prophylaxis on sensory recovery and postoperative pain following orthognathic surgery: a triple-blind randomised controlled trial and biochemical analysis. Int $J$ Oral Maxillofac Surg. 2019.

29. Su X, Wang DX. Improve postoperative sleep: what can we do? Current opinion in anesthesiology. 2018;31(1):83-8.

30. Bjurstrom MF, Irwin MR. Perioperative Pharmacological Sleep-Promotion and Pain Control: A Systematic Review. Pain Pract. 2019;19(5):552-69.

31. Zhang J, Wang Y, Xu H, Yang J. The Influence of Melatonin on Sleep Quality After Laparoscopic Cholecystectomy: A Meta-Analysis of Randomised Controlled Trials. Surg Laparosc Endosc Percutan Tech. 2019;29(1):1-6.

32. Kirksey MA, Yoo D, Danninger T, Stundner O, Ma Y, Memtsoudis SG. Impact of Melatonin on Sleep and Pain After Total Knee Arthroplasty Under Regional Anesthesia With Sedation: A Double-Blind, Randomised, Placebo-Controlled Pilot Study. J Arthroplasty. 2015;30(12):2370-5.

33. Ip HY, Abrishami A, Peng PW, Wong J, Chung F. Predictors of postoperative pain and analgesic consumption: a qualitative systematic review. Anesthesiology. 2009;111(3):657-77.

34. Khezri MB, Merate $H$. The effects of melatonin on anxiety and pain scores of patients, intraocular pressure, and operating conditions during cataract surgery under topical anesthesia. Indian $\mathrm{J}$ Ophthalmol. 2013;61(7):319-24.

35. Hansen MV, Halladin NL, Rosenberg J, Gogenur I, Moller AM. Melatonin for pre- and postoperative anxiety in adults. The Cochrane database of systematic reviews. 2015(4):CD 009861.

36. de Oliveira Tatsch-Dias M, Levandovski RM, Custodio de Souza IC, Gregianin Rocha M, Magno Fernandes PA, Torres IL, et al. The concept of the immune-pineal axis tested in patients undergoing an 
abdominal hysterectomy. Neuroimmunomodulation. 2013;20(4):205-12.

37. Hugoy $T$, Lerdal A, Rustoen $T$, Oksholm T. Predicting postoperative fatigue in surgically treated lung cancer patients in Norway: a longitudinal 5-month follow-up study. BMJ Open. 2019;9(9):e028192.

38. Wodlin NB, Nilsson L, Arestedt K, Kjolhede P, Group GS. Mode of anesthesia and postoperative symptoms following abdominal hysterectomy in a fast-track setting. Acta Obstet Gynaecol Scand. 2011;90(4):369-79.

\section{Tables}

Table 1 Demographic data and patients' characteristics

\begin{tabular}{llll} 
Patient characterisitics & $\begin{array}{l}\text { P group } \\
(\mathbf{n = 2 7 )}\end{array}$ & $\begin{array}{l}\text { M group } \\
(\mathbf{n}=\mathbf{2 7})\end{array}$ & p-value \\
\hline Age (year) & $42.85 \pm 5.16$ & $44.93 \pm 4.13$ & 0.11 \\
Weight (kg) & $59.07 \pm 8.88$ & $60.0 \pm 7.86$ & 0.69 \\
BMI (kg/m2) & $23.77 \pm 3.59$ & $24.48 \pm 3.22$ & 0.45 \\
ASA PS I/II & $23 / 4$ & $22 / 5$ & 0.72 \\
Diagnosis & & & 0.49 \\
Myoma uteri & 20 & 21 & \\
Adenomyosis & 6 & 4 & \\
ovarian cyst & 1 & 2 & \\
Operation & & & \\
TAH & 15 & 14 & \\
TAH and SO & 12 & 13 &
\end{tabular}

Results showed in mean \pm S.D., ASA PS $=$ The American Society of Anesthesiologists physical status, $\mathrm{BMI}=$ Body Mass Index, $\mathrm{TAH}=$ Trans Abdominal Hysterectomy, $\mathrm{SO}=$ Salpingo-oopherectomy

Table 2 Intraoperative outcomes 


\begin{tabular}{llll} 
Variable & $\begin{array}{l}\text { P group } \\
(\mathbf{n = 2 7 )}\end{array}$ & $\begin{array}{l}\text { M group } \\
(\mathbf{n = 2 7})\end{array}$ & p-value \\
\hline Dose of bupivacaine (mg) & $18.25 \pm 1.05$ & $18.75 \pm 0.8$ & 0.18 \\
Anesthetic level (T4/T6) & $18 / 9$ & $22 / 5$ & 0.19 \\
Sedation requirement (Yes/No) & $11 / 16$ & $16 / 11$ & 0.27 \\
Propofol dose & $90.37 \pm 25.47$ & $138.15 \pm 27.61$ & 0.21 \\
Skin incision & & & \\
(Low midline/Pfennenstiel) & $4 / 23$ & $8 / 19$ & 0.32 \\
Duration of surgery (min) & $105.56 \pm 19.23$ & $103.52 \pm 31$ & 0.77 \\
Blood loss (mL) & $220.37 \pm 23.90$ & $247.04 \pm 29.89$ & 0.49 \\
Intraoperative events (Yes/no) & $18 / 9$ & $20 / 7$ & 0.83 \\
Hypotension need treatment & $16(59.3 \%)$ & $18(66.7 \%)$ & 0.64 \\
Ephedrine dose (mg) & $5 \pm 6.17$ & $6.15 \pm 6.7$ & 0.51 \\
Bradycardia & 0 & 0 & \\
Nausea/Vomiting & $2(7.4 \%)$ & $2(7.4 \%)$ & 0.56
\end{tabular}

Results showed in mean \pm S.D or $\mathrm{n}(\%)$

Table 3 Analgesic outcomes, other subjective scores and adverse effects 


\begin{tabular}{|c|c|c|c|}
\hline Outcomes & $\begin{array}{l}\text { P group } \\
(n=27)\end{array}$ & $\begin{array}{l}M \text { group } \\
(n=27)\end{array}$ & $p$-value \\
\hline Morphine consumption in $24 \mathrm{hr}$ (mg) & $42.63 \pm 8.21$ & $33.04 \pm 10.42$ & $<0.01^{\star}$ \\
\hline \multicolumn{4}{|l|}{ VAS pain score $(\mathrm{mm})$} \\
\hline T0 & $23.41 \pm 4.62$ & $8.07 \pm 3.39$ & $0.001^{\star}$ \\
\hline T1 & $41.67 \pm 9.13$ & $35.26 \pm 5.88$ & 0.19 \\
\hline T6 & $56.74 \pm 8.87$ & $48.89 \pm 7.94$ & 0.21 \\
\hline T24 & $34.37 \pm 6.56$ & $29.26 \pm 4.91$ & 0.12 \\
\hline VAS Satisfaction score & $7.78 \pm 1.05$ & $8.56 \pm 1.25$ & $0.02^{\star}$ \\
\hline \multicolumn{4}{|l|}{ Preoperative } \\
\hline Level of anxiety & $8.63 \pm 3.48$ & $8.11 \pm 4.09$ & 0.62 \\
\hline VAS sleep quality & $26.89 \pm 26.34$ & $23.81 \pm 26.41$ & 0.67 \\
\hline VAS fatigue level & $2.19 \pm 2.15$ & $1.85 \pm 2.45$ & 0.59 \\
\hline VAS general well being & $20.70 \pm 21.65$ & $22.63 \pm 23.11$ & 0.75 \\
\hline \multicolumn{4}{|l|}{ Postoperative at $24 \mathrm{hr}$} \\
\hline Level of anxiety & $5.44 \pm 1.37$ & $5.00 \pm 2.00$ & 0.35 \\
\hline VAS sleep quality & $57.93 \pm 21.08$ & $49.33 \pm 21.02$ & 0.14 \\
\hline VAS fatigue & $5.15 \pm 1.85$ & $3.30 \pm 2.22$ & $0.002 *$ \\
\hline VAS general well being & $49.78 \pm 14.87$ & $31.59 \pm 24.14$ & $0.002^{\star}$ \\
\hline Surgical complications & 0 & 0 & \\
\hline VAS Nausea/Vomiting in $24 \mathrm{hr}$ after surgery & $3.7 \pm 2.52$ & $4.1 \pm 2.67$ & 0.56 \\
\hline
\end{tabular}

Results showed in mean \pm S.D or $\mathrm{n}(\%)$

\section{Figures}




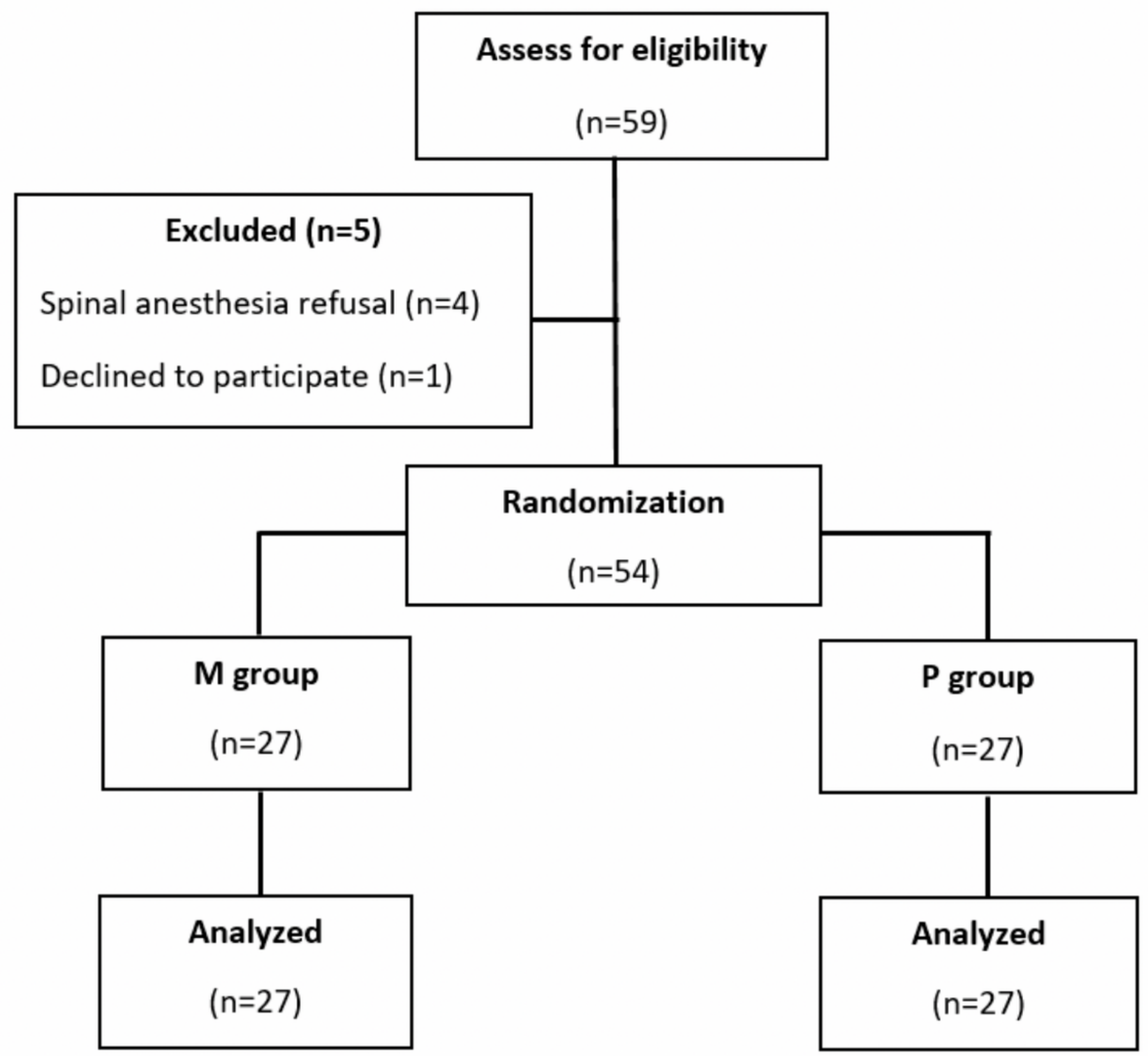

Figure 1

CONSORT diagram 